\title{
Occurrence of a left ventricular third heart sound in incompetent mitral heterografts
}

\author{
Mamdouh El Gamal and Donald R. Smith \\ From The Cardiac Department, Leeds General Infirmary, Leeds
}

Three cases of severe mitral incompetence after heterograft aortic valve replacement are described. In all cases an early third heart sound was recorded on the phonocardiograms. In two cases a prominent early diastolic peak was also recorded on the apex cardiograms. These findings do not support the widely held view that the third heart sound is produced by sudden tautening of the mitral valve structures during the rapid filling phase of the left ventricle.

The mechanisms responsible for the normal or pathological third heart sounds are uncertain, and as a result various theories have been proposed since the beginning of this century. The present report describes three patients who developed severe mitral incompetence after valve replacement with aortic heterografts. In each an early third heart sound was audible over the apex and recorded on the phonocardiograms. In two of the cases a prominent diastolic peak was recorded on the apex cardiogram, corresponding in timing to the third heart sound point on the phonocardiogram, thus confirming that it is of left ventricular origin.

The occurrence of a third heart sound in these patients is incompatible with the theory that the third sound is caused by sudden tautening of the mitral valve cusps and chordae, as the latter structures had been previously excised at operation. As controversy still exists concerning the genesis of the third heart sound, it is thought worth while to record these observations.

\section{Methods}

Phonocardiograms were obtained pre- and postoperatively in Case I, post-operatively only in Cases 2 and 3. Medium frequency phonocardiograms were recorded with New Electronics Products Recorder apparatus. Apex cardiograms were recorded before and after operation in Case I, after operation only in Case 3, using a Phillips displacement transducer.

\section{Case reports}

Case I A 34-year-old man presented complaining of progressive shortness of breath on mild

Received II November 1969. exertion, and attacks of paroxysmal nocturnal dyspnoea. He gave a past history of rheumatic chorea at the age of 13 .

On examination his venous pressure was normal and he was in sinus rhythm. Biventricular hypertrophy was apparent. The auscultatory findings indicated a mixed mitral lesion, pulmonary hypertension, and very mild aortic incompetence (pre-operative phonocardiogram Fig. I, apex cardiogram Fig. 2).

On screening, heavy calcification of the mitral valve was seen. At cardiac catheterization his systolic right ventricular pressure was $80 \mathrm{~mm} . \mathrm{Hg}$ and the mean left atrial pressure was $35 \mathrm{~mm}$. $\mathrm{Hg}$. No systolic gradient was detected across the aortic valve and an aortic root angiogram revealed only minimal aortic incompetence.

At operation on Io April 1967 the mitral valve was found to be heavily calcified and stenotic, the orifice being $1.5 \mathrm{~cm}$. The entire mitral valve and chordae tendineae were excised, and an aortic heterograft previously sutured to a Teflon tube and ring was inserted (Ionescu, Mashhour, and Wooler, 1968). Post-operative recovery was uneventful. He was subsequently readmitted as an emergency on 9 July with a 3-week history of severe breathlessness.

On auscultation, he had a loud mitral pansystolic murmur and a third heart sound (postoperative phonocardiogram Fig. 3, apex cardiogram Fig. 4).

On 25 July he underwent a second operation and it was found that the mitral heterograft had separated from the Teflon ring resulting in severe mitral incompetence. The valve was replaced with another heterograft, but the patient died the following day from an arrhythmia.

At necropsy the second mitral heterograft was found to be fully competent.

Case 2 A 54-year-old man gave a past history of four attacks of rheumatic fever from the age of Io to 20 years. He presented complaining of 
increasing breathlessness and attacks of nocturnal dyspnoea.

On examination he was in atrial fibrillation, the cardiac signs being those of gross mitral incompetence.

On 30 October 1967 he was operated on and a destroyed incompetent mitral valve was found. This was excised with its chordae and replaced with an aortic heterograft mounted on a titanium frame.

After operation he had a soft mitral pansystolic murmur and was breathless on exertion. His condition gradually deteriorated and by August I 968 he was in congestive heart failure. On auscultation he had an apical pansystolic murmur and a third heart sound (phonocardiogram Fig. 5).

On 27 August 1968 a second operation was performed. It was found that the heterograft had two holes between the ring and annulus where sutures had pulled through. This valve was replaced with a No. 4 Starr-Edwards mitral prosthesis.

After a stormy post-operative period, the patient slowly improved and was well when last seen at a follow-up clinic.

Case 3 A 36-year-old man gave a past history of rheumatic fever at the age of 12 and bacterial endocarditis at the age of 25 . In October 1967 he was complaining of shortness of breath on mild exertion, attacks of nocturnal dyspnoea, and ankle swelling.

On examination his venous pressure was raised $6 \mathrm{~cm}$. and he was in atrial fibrillation. The heart was enlarged, the auscultatory findings being those of mitral stenosis and incompetence and pulmonary hypertension.

Operation was undertaken on 4 December 1967 , a mainly stenotic but destroyed mitral valve being found. This was excised and replaced with an aortic heterograft mounted on a titanium ring.

He made a good recovery and was subsequently catheterized in March 1968. The systolic right ventricular pressure was then $30 \mathrm{~mm} . \mathrm{Hg}$, and the mean wedge pressure was II $\mathrm{mm}$. $\mathrm{Hg}$. A left ventricular angiogram was performed and this did not reveal any mitral incompetence.

In May I968 a soft mitral pansystolic murmur was heard for the first time, but he was then free from symptoms. He was readmitted to hospital on 8 September 1969 with a history of increasing shortness of breath, orthopnoea, and ankle swelling of 8 weeks' duration.

On examination his venous pressure was raised $8 \mathrm{~cm}$., the heart was enlarged, and on auscultation over the apex, he had a loud pansystolic murmur, and a third heart sound (phonocardiogram, Fig. 6, apex cardiogram, Fig. 7). At re-operation on 7 October 1969 , it was found that one commissure had detached from the aortic wall, resulting in severe mitral incompetence. The defective valve was excised, and replaced with a fascia lata graft attached to a scallop-shaped titanium frame covered with 'Dacron' velour (Ionescu and Ross, 1969). After this he made good progress, and post-operatively has normal heart sounds.
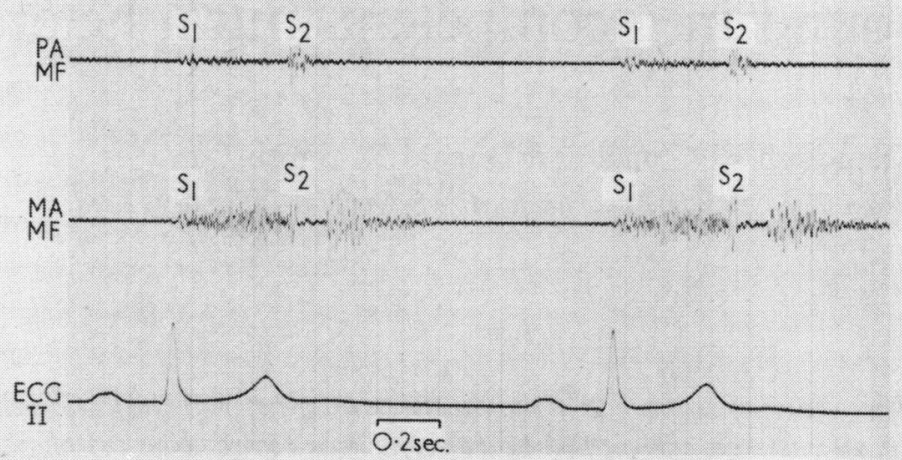

FIG. I Pulmonary area medium frequency

$(P A / M F)$ and mitral area medium frequency

$(M A / M F)$ phonocardiograms, and lead II

electrocardiogram (ECGII). The mitral phono-

cardiogram records the pansystolic murmur of

mitral regurgitation. The diastolic murmur has

a loud onset.

FIG. 2 Apex cardiogram ( $A D C$ ), lower

sternal edge medium frequency ( $L S E / M F)$

phonocardiogram, and lead II electrocardio-

gram (ECGII). The apex cardiogram shows a

small rapid filling wave peak $\left({ }_{3} P\right)$, followed

by a slow filling wave ( $S F W)$.

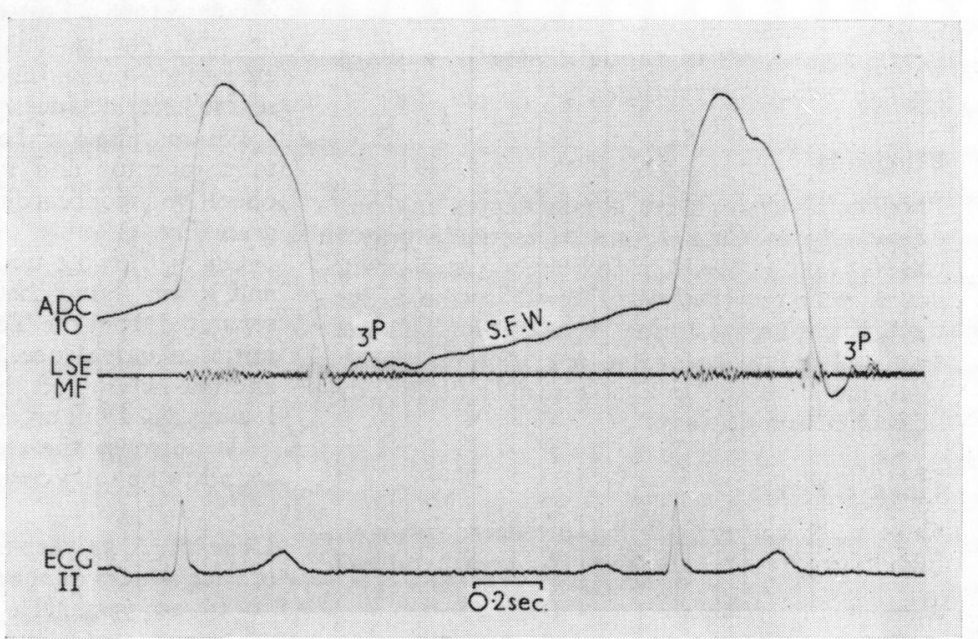




\section{Discussion}

Theories regarding the production of third heart sounds are broadly divisible into two categories.

(I) That the sound is produced by an increased rate of left ventricular filling, the rapidly dilating ventricle reaching a point where distension is limited by the fibrous resistance of its walls. The sharp check of the distending ventricle then initiates the vibrations audible as the third sound (Potain, 1900; Crevasse et al., 1962; Kuo et al., 1957).

(2) That the sound originates in the structures of the mitral valve itself. Gibson (1907) and Thayer (1909) suggested that early closure of the mitral valve cusps produced the vibrations of the third sound. An alternative explanation, favoured by Dock (1959), Nixon (196I), and Fleming (1969), is that the sound arises from the mitral valve cusps, chordae tendineae, and papillary muscles, these structures being set into vibration towards the end of the rapid filling phase of the left ventricle. As the left ventricle fills, the apex descends and the atrioventricular ring ascends, thus elongating the ventricle. Nixon (196I) and Fleming (1969) suggest that the process of elongation of the ventricle is checked by the mitral valve structures coming under tension, and that vibrations induced by the sudden tautening of the mitral valve, chordae tendineae, and papillary muscles are heard as the third heart sound. The cases described in the present report argue against the proposals of Nixon (I96I) and Fleming (I969), for third heart sounds have been shown in patients developing mitral incompetence in whom the continuity of the mitral valve structures had been severed at an earlier operation. Any mechanism involving tautening of the chordae tendineae and/or papillary muscles seems to be excluded by these findings.

Fleming (1969) reported the complete absence of apical retraction in early diastole in apex cardiograms in 23 cases of mitral valve replacement with Starr-Edwards prostheses. He uses this finding to argue in favour of the proposal that the retraction of the apex in early diastole, which occurs coincident in timing with the third heart sound, is also due to the tautening of the mitral valve structures. The apex cardiograms of Cases I and 3, with incompetent mitral heterografts, show prominent diastolic peaks, indicating that this can occur despite surgical removal of the chordae tendineae.

From the findings presented here, we believe that neither the third heart sound as heard, nor the apical retraction as demonstrated on apex cardiograms can be adequately

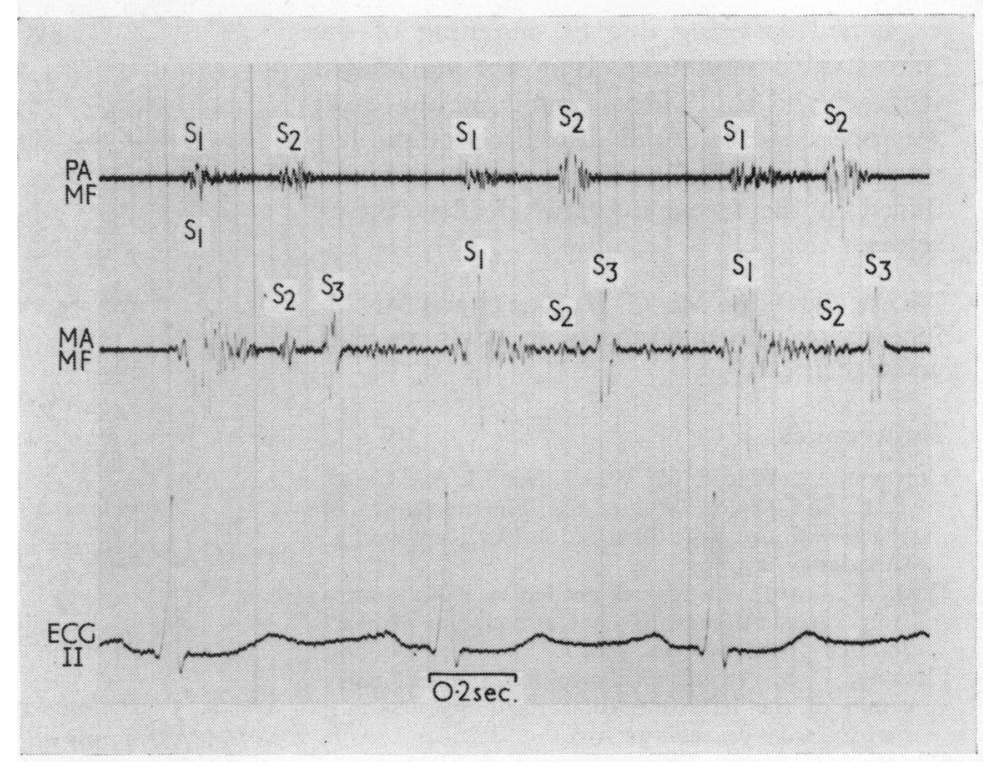

FIG. 3 Pulmonary area medium frequency

$(P A / M F)$ and mitral area medium frequency

(MA/MF) phonocardiograms, and lead II electrocardiogram (ECGII). The mitral phonocardiogram records a pansystolic murmur of loud onset, and a third heart sound $0 \cdot 1-0 \cdot 12$ sec. after aortic valve closure.

FIG. 4 Apex cardiogram ( $A D C$ ), lower sternal edge medium frequency ( $L S E / M F)$ phonocardiogram, and lead II electrocardiogram (ECGII). The apex cardiogram shows a much accentuated ' $a$ ' wave and a prominent third sound point $(3 P) O \cdot I-O \cdot I 2$ sec. after aortic valve closure.

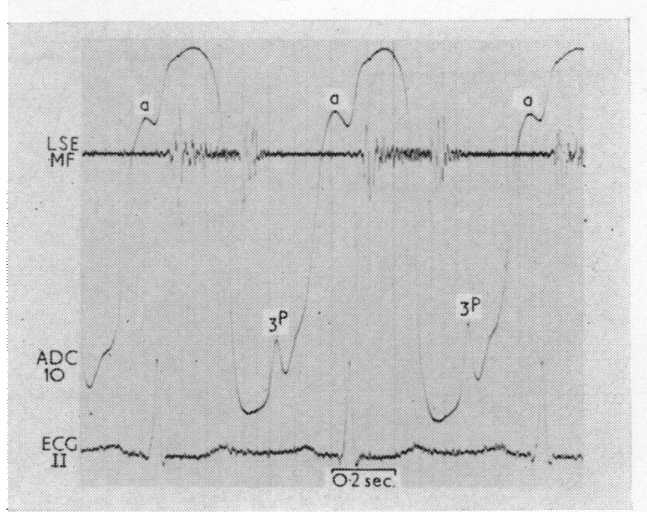


explained as being due to tautening of the mitral valve structures. It is not yet known whether the third heart sound in our cases was produced by rapid distension of the left ventricular muscle itself or by vibrations induced in the free heterograft leaflets themselves.

We are grateful to Mr. G. H. Wooler and Mr. M. Ionescu for the opportunity to study patients under their care.

\section{References}

Crevasse, L., Wheat, M. W., Wilson, J. R., Leeds, R. F., and Taylor, W. J. (1962). The mechanism of the generation of the third and fourth heart sounds. Circulation, 25, 635 .

Dock, W. (1959). The forces needed to evoke sounds from cardiac tissues, and the attenuation of heart sounds. Circulation, 19, 376.

Fleming, J. S. (1969). Evidence for a mitral valve origin of the left ventricular third heart sound. British Heart fournal, 31, 192.

Gibson, A. G. (1907). The significance of a hitherto undescribed wave in the jugular pulse. Lancet, 2, 1380.

Ionescu, M. I., Mashhour, Y. A. S., and Wooler, G. H. (1968). Reconstructed heterograft aortic valves for human use. Preparation and surgical implantation for mitral, aortic, and tricuspid replacement. Thorax, 23, 221.

- , and Ross, D. N. (1969). Heart-valve replacement with autologous fascia lata. Lancet, 2, 335.

Kuo, P. T., Schnabel, T. G., Blakemore, W. S., and Whereat, A. F. (1957). Diastolic gallop sounds; the mechanism of production. Fournal of Clinical Investigation, 36, 1035 .

Nixon, P. G. F. (1961). The third heart sound in mitral regurgitation. British Heart fournal, 23, 677.

Potain, P. C. E. (1900). Les bruits de galop. Semaine Médicale, 20, 175.

Thayer, W. S. (1909). Further observations on the third heart sound. Archives of Internal Medicine, 4, 297.

FIG. 6 Pulmonary area medium frequency $(P A / M F)$ and mitral area medium frequency $(M A / M F)$ phonocardiograms, and lead II electrocardiogram (ECGII). The mitral phonocardiogram records a pansystolic murmur, and a third heart sound $0 \cdot 1 \mathrm{sec}$. after aortic valve closure.

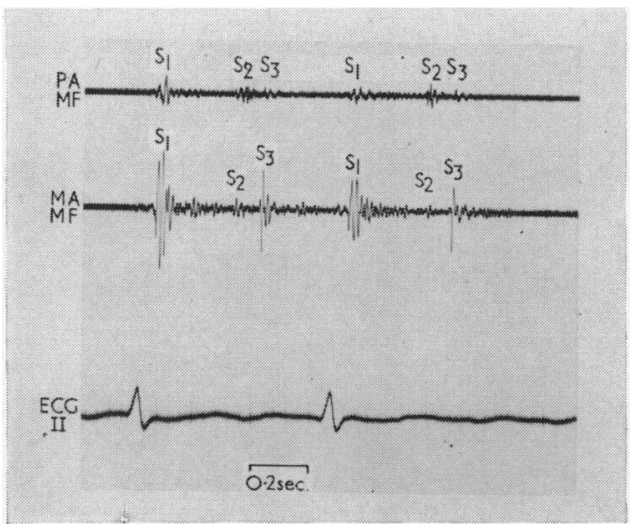

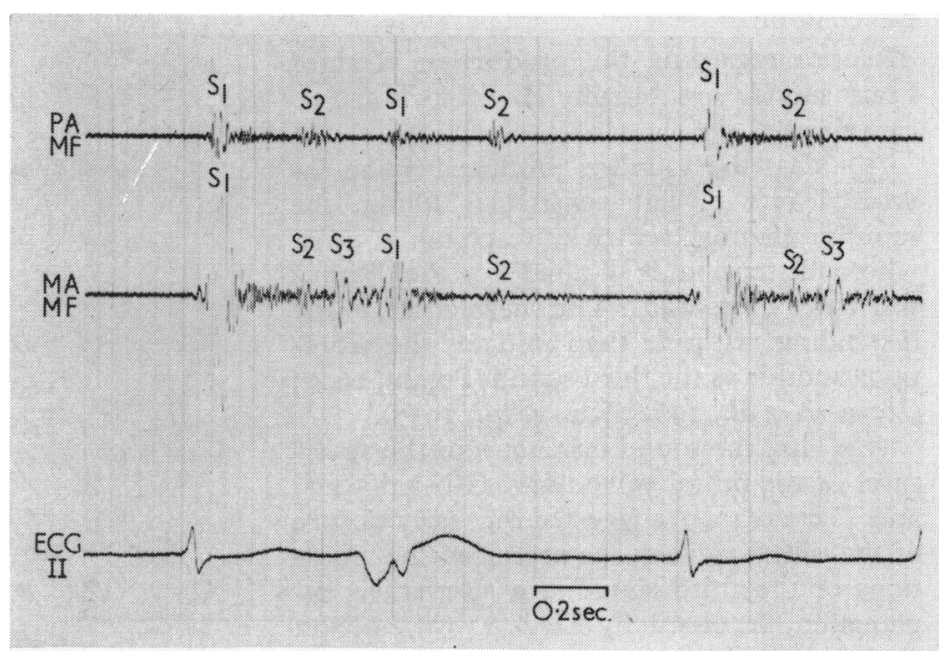

FIG. 5 Pulmonary area medium frequency $(P A / M F)$ and mitral area medium frequency $(M A / M F)$ phonocardiograms, and lead II electrocardiogram (ECGII). The mitral phonocardiogram records a pansystolic murmur and a third heart sound $0 \cdot 1$ sec. after aortic valve closure.

FI G. 7 Apex cardiogram ( $A D C I 0$ ), lower sternal edge medium frequency ( $L S E / M F$ ) phonocardiogram, and lead II electrocardiogram (ECGII). The apex cardiogram shows a prominent third sound point $(3 P) 0.12 \mathrm{sec}$. after aortic valve closure coinciding with the third sound on the phonocardiogram.

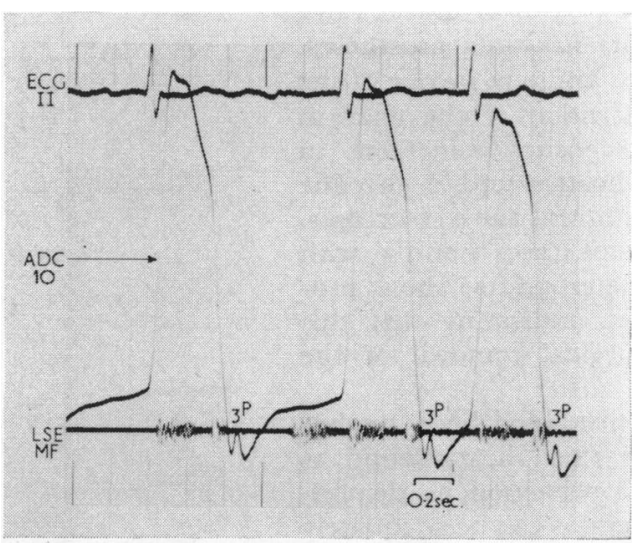

